\title{
D-Cycloserine Augmentation of Exposure Therapy for Post-Traumatic Stress Disorder: A Pilot Randomized Clinical Trial
}

\author{
JoAnn Difede ${ }^{*, 1,2}$, Judith Cukor ${ }^{1,2}$, Katarzyna Wyka', Megan Olden ${ }^{1,2}$, Hunter Hoffman ${ }^{3}$, \\ Francis S Lee ${ }^{1,2}$ and Margaret Altemus ${ }^{1,2}$ \\ 'Department of Psychiatry, Weill Cornell Medical College, New York, NY, USA; ${ }^{2}$ NewYork-Presbyterian Hospital, New York, NY, USA; \\ ${ }^{3}$ Department of Mechanical Engineering, Human Interface Technology Laboratory, and Human Photonics Laboratory, University of Washington, \\ Seattle, WA, USA
}

\begin{abstract}
Viewing post-traumatic stress disorder (PTSD) as a disorder of emotional learning, this study used a cognitive enhancer synergistically with virtual reality exposure (VRE) therapy for the treatment of PTSD. The main objective was to determine if a novel pharmacotherapy, D-cycloserine (DCS), enhanced the efficacy of the psychotherapy. Pre-clinical studies suggest that when fear extinction occurs during DCS administration, neuroplasticity may be enhanced. VRE therapy is a particularly promising format to test the hypothesis that DCS enhances extinction learning, as sensory fear cues are standardized across patients. In a pilot randomized, double-blind, placebocontrolled trial, $100 \mathrm{mg}$ of DCS or placebo was administered $90 \mathrm{~min}$ before each weekly VRE session, to ensure peak plasma concentrations during the sessions in 25 patients with chronic PTSD. The primary outcome measure was the Clinician Administered PTSD Scale (CAPS). Secondary outcome measures included the Beck Depression Inventory-II and the State-Trait Anger Expression Inventory-2. Assessments occurred at pre-treatment, following sessions 3, 6, 10, post-treatment, and at 6 months. The difference in CAPS between the VRE-DCS $(n=13)$ and VRE-placebo $(n=12)$ groups increased over time beginning at 6 weeks, with medium to large between-group effect sizes immediately post-treatment and 6 months later $(d=0.68$ and $d=1.13$, respectively). A similar pattern was observed for depression, anger expression, and sleep. PTSD remission rates were significantly greater for the VRE-DCS group (46\% vs $8 \%$ at post-treatment; $69 \%$ vs 17\% at 6 months). Patients in the VRE-DCS group showed earlier and greater improvement in PTSD symptoms compared with the VRE-placebo group. These results suggest a promising new treatment for PTSD.

Neuropsychopharmacology (2014) 39, I052-1058; doi:I0.1038/npp.20 I3.3 I7; published online II December 2013
\end{abstract}

Keywords: post-traumatic stress disorder; exposure therapy; virtual reality; D-cycloserine; translational research; terrorism

\section{INTRODUCTION}

Effective treatments for post-traumatic stress disorder (PTSD) remain elusive (Institute of Medicine (IOM), 2008). Yet, PTSD is a significant public health problem with an estimated $8-9 \%$ of the population at risk for at least one episode during their lifetime (Hidalgo and Davidson, 2000; Kessler et al, 1995; Yule, 2001). PTSD is associated with multiple adverse outcomes, including psychiatric comorbidity, marital discord, medical illness (Dedert et al, 2010), absenteeism (Alonso et al, 2011), suicide (Kessler, 2000), and an estimated annual productivity loss of approximately \$3 billion (Kessler, 2000).

One novel treatment approach follows from the hypothesis that PTSD is a disorder of emotional learning (Foa and

*Correspondence: Dr J Difede, Department of Psychiatry, Weill Cornell Medical College, 525 East 68th Street, Box 200, New York, NY | 0065, USA, Tel: + | 212746 3079, Fax: + | 212746 07|9,

E-mail: jdifede@med.cornell.edu

Received 27 June 2013; revised 15 October 2013; accepted 4 November 2013; accepted article preview online 12 November 2013
Kozak, 1986). PTSD is the only psychiatric disorder that requires the occurrence of an external event as a prerequisite to diagnosis. This event provides the context for learning. Across species, pairing a neutral stimulus with an aversive one leads to the learning of a conditioned fear response. In humans with PTSD, the matrix of sensory stimuli embedded in the traumatic memory serve as cues that evoke a conditioned fear response in the absence of the original trauma (the unconditioned aversive stimuli). This conditioned fear response manifests as avoidance of traumaassociated cues-including thoughts, feelings, or sensory (eg, olfactory) reminders-and the experience of emotional distress when faced with these reminders. A conditioned fear response can be initially adaptive, but it should extinguish when the conditioned cues are no longer accompanied by actual risk of danger (Foa and Kozak, 1986). Individuals with PTSD have not learned that the stimuli associated with their trauma are now safe. Thus, PTSD may manifest from a persisting conditioned fear response independent of the original trauma and difficulty learning that stimuli previously associated with a trauma are no longer so. 
If PTSD results from disordered learning, then developing strategies to correct these errors of emotional learning and memory may offer key avenues for recovery. In theory, any intervention that facilitates extinction learning in humans could be efficacious. Animal and human studies robustly demonstrate that fear is extinguished experimentally by repeatedly presenting the conditioned stimulus in the absence of the aversive stimulus. In humans, this model translates into repeatedly re-experiencing the traumatic memory in a safe environment (absence of the aversive stimuli) until the fear is extinguished. This process is hypothesized to be the mechanism of action in exposure therapy, the treatment with the strongest empirical evidence for PTSD (Foa et al, 2000; Institute of Medicine (IOM), 2008).

Novel pharmacotherapeutics that augment the extinction learning process that may occur during exposure therapy offer a unique approach to improve treatment response. D-cycloserine (DCS) (Seromycin) is a partial agonist at the $\mathrm{N}$-methyl-D-aspartate (NMDA) receptor, a member of the glutamate receptor family, which has an essential role in mediating learning and memory. Both fear learning and extinction are blocked by antagonists at the glutamatergic NMDA receptor (Zimmerman and Maren, 2010). Preclinical research has demonstrated that DCS facilitates extinction learning and memory in rodent models (Davis et al, 2006; Ledgerwood et al, 2003; Walker et al, 2002). Recently, DCS combined with imaginal or virtual reality exposure (VRE) therapy has been shown to reduce length of treatment for some anxiety disorders, including acrophobia (Ressler et al, 2004), social phobia (Guastella et al, 2008; Hofmann et al, 2006), and panic disorder (Otto et al, 2010), which, like PTSD, include learned avoidance and cued emotional distress as salient symptoms.

This study examined the potential of DCS to enhance VRE therapy in the treatment of chronic WTC-related PTSD. VRE utilizes a multi-sensory virtual environment to enhance emotional engagement during exposure. Thus, the patient relates his trauma narrative repeatedly, but instead of closing his eyes as in imaginal exposure, he is immersed in a virtual environment and exposed to visual, auditory, and haptic cues that evoke the traumatic memory. Our previous studies have documented the efficacy of VRE therapy for the treatment of WTC-related PTSD (Difede et al, 2007). Although other effective variations of exposure therapy exist (Institute of Medicine (IOM), 2008), VRE therapy was chosen because of the potential to standardize sensory stimuli across participants.

We hypothesized that participants in the VRE-DCS group would show a significantly earlier and larger decrease in PTSD symptoms compared with participants in the VREplacebo group, and that these differences would be maintained at 6 months.

\section{MATERIALS AND METHODS}

\section{Participants}

Eligible participants were English-speaking adults aged 1870 years who were in good health and had PTSD following exposure to the WTC attacks. Exclusion criteria were: substance dependence, active suicidal or homicidal idea- tion, significant health impairment, current use of oral anticoagulant medication, anti-tuberculosis medications, history of seizures, or hypersensitivity to DCS. Participants on psychotropic medications (which included Paxil, Xanax, Klonopin, Lexapro, Prozac, Wellbutrin, Effexor, Ativan, Nortriptyline, Celexa, Cymbalta, and Zoloft) had to be on a stable dose for at least 2 months before enrollment and maintain their medication regimen throughout the study. Participants were recruited between 2005 and 2011 by publicizing the study in medical centers and the general community. This study was approved by the Weill Cornell Medical College Institutional Review Board and subjects provided written informed consent.

\section{Randomization}

Of 55 candidates evaluated, 30 were ineligible or declined participation (Supplementary Figure S1). The remaining 25 participants were randomized to receive VRE therapy combined with either $100 \mathrm{mg}$ DCS ( $n=13$; VRE-DCS group) or placebo ( $n=12$; VRE-placebo group). Twenty-two participants completed the study intervention and 6-month follow-up. Three participants dropped out (after sessions 3, 6 , and 7); all were in the VRE-placebo group. The majority of participants were white $(n=21,84 \%)$, male $(n=19$, $76 \%)$, college graduates $(n=17,68 \%)$ with a mean age of 45.84 ( $\mathrm{SD}=10.50$, range: $25-70$ years) who were either married or lived with their significant other $(n=15,60 \%)$. Eleven participants were from occupations-at-risk for PTSD (4 firefighters, 6 police, and 1 EMT/paramedic), and 14 participants were civilians. There were no significant differences between treatment groups on any demographic or study variables (Table 1), including patients' expectations regarding treatment effectiveness.

\section{Measures}

The primary outcome measure was the Clinician Administered PTSD Scale (CAPS; Blake et al, 1998). Comorbid psychiatric diagnoses were assessed using the Structured Clinical Interview for the DSM-IV (SCID-I; First et al, 1997). Self-report measures included the PTSD Checklist (PCL; Weathers et al, 1993), Beck Depression Inventory-second edition (BDI-II; Beck et al, 1996), and State-Trait Anger Expression Inventory-2 (STAXI-2; Spielberger, 1999). Treatment expectancy was assessed weekly with a four-item Likert scale measure used in prior PTSD treatment outcome studies (Difede et al, 2007).

\section{Medication}

DCS is an FDA-approved antibiotic that has been previously used in clinical trials as a cognitive enhancer. $100 \mathrm{mg}$ DCS capsules and matching placebo capsules containing lactose were prepared. Medication was administered double-blind; the research pharmacy oversaw randomization. Participants took one pill $90 \mathrm{~min}$ before their weekly exposure session (sessions 2-11). Compliance was assessed by verbal inquiry about the exact timing of medication ingestion. Less than $1 \%$ of the time ( 2 of 255 sessions) participants reported forgetting to take the study medication; in these instances 
Table I Participant Sociodemographic Characteristics

\begin{tabular}{|c|c|c|c|}
\hline Variable & $\begin{array}{l}\text { VRE-DCS } \\
(n=13)\end{array}$ & $\begin{array}{l}\text { VRE-placebo } \\
\quad(n=12)\end{array}$ & $\begin{array}{c}\text { Test of } \\
\text { significance }\end{array}$ \\
\hline Age M (SD) & $47.77(11.92)$ & $43.75(8.72)$ & $\begin{array}{l}t=-0.96 \\
p=0.350\end{array}$ \\
\hline \multicolumn{4}{|l|}{ Gender } \\
\hline Male & 8 & 11 & $\begin{array}{l}\chi^{2}=3.11 \\
p=0.078\end{array}$ \\
\hline Female & 5 & I & \\
\hline \multicolumn{4}{|l|}{ Education } \\
\hline $\begin{array}{l}\text { Some or no high } \\
\text { school }\end{array}$ & 0 & 0 & $\begin{array}{l}\chi^{2}=2.00 \\
p=0.573\end{array}$ \\
\hline High school & 2 & I & \\
\hline Some college & 3 & 2 & \\
\hline College & 8 & 9 & \\
\hline \multicolumn{4}{|l|}{ Marital status } \\
\hline Cohabitating & । & 2 & $\begin{array}{l}\chi^{2}=2.63 \\
p=0.452\end{array}$ \\
\hline Separated/divorced & 4 & I & \\
\hline Married & 5 & 7 & \\
\hline Single & 3 & 2 & \\
\hline \multicolumn{4}{|l|}{ Ethnicity } \\
\hline Caucasian & 10 & 11 & $\begin{array}{l}\chi^{2}=1.34 \\
p=0.511\end{array}$ \\
\hline Black & । & 0 & \\
\hline Hispanic & 2 & । & \\
\hline Major depression & 9 & 7 & $\begin{array}{l}\chi^{2}=0.32 \\
p=0.571\end{array}$ \\
\hline Trauma history & 13 & 9 & $\begin{array}{l}\chi^{2}=3.69 \\
p=0.055\end{array}$ \\
\hline $\begin{array}{l}\text { Concurrent } \\
\text { medication use }^{\mathrm{a}}\end{array}$ & 9 & 5 & $\begin{array}{l}\chi^{2}=1.92 \\
p=0.165\end{array}$ \\
\hline $\begin{array}{l}\text { VR sessions } \\
\text { completed }\end{array}$ & $11.00(1.00)$ & $9.33(3.34)$ & $\begin{array}{c}t=-1.62 \\
p=0.121\end{array}$ \\
\hline
\end{tabular}

aVRE-DCS: eight antidepressants, four other psychiatric medications, four both; VRE-placebo: four antidepressants, five other psychiatric medications, four both.

the session was rescheduled. A side-effect questionnaire was completed at each session; no side effects were reported.

We chose weekly dosing, administered $90 \mathrm{~min}$ before exposure sessions, as this regimen has been effective in multiple animal and human fear extinction studies (Ledgerwood et al, 2003; Ressler et al, 2004; Walker et al, 2002) and because daily, longer-term dosing appears to reduce the cognitive enhancing effects of DCS (Quartermain et al, 1994), possibly due to desensitization of the NMDA receptor over time (Boje et al, 1993). The chosen dosing regimen allowed sufficient time for the medication to be absorbed into the bloodstream and for the session to be completed while circulating levels of the medication were still high. Prior human research has shown that peak cerebrospinal fluid concentrations occur
$2 \mathrm{~h}$ following a single oral dose of $250 \mathrm{mg}$ DCS (Baron et al, 1956), and that peak blood levels occur 3-4 h after dosing. Previously, DCS was given either 2-4h (Ressler et al, 2004), or $1 \mathrm{~h}$ (Hofmann et al, 2006), before each exposure session. Similarly, both the 2-week acrophobia study (Ressler et al, 2004) and 5-week social anxiety study supported the use of a weekly dosing strategy (Hofmann et al, 2006). The choice of dosing regimen for our studies was based on the Ressler et al (2004) study, which showed that there was no difference in efficacy between the 50 and $500 \mathrm{mg}$ dose of DCS on enhancing exposure therapy outcomes. We chose to use a single dose (ie, $100 \mathrm{mg}$ ) within the lower end of the dosing range of this initial DCS study to minimize the likelihood of side effects.

\section{Study Intervention}

The VRE treatment protocol consisted of 12 weekly, 90-min sessions (for a description of the treatment, see Difede et al, 2007). Sessions 2-11 focused on the VRE therapy, during which participants recounted their trauma in first-person present tense, with as many sensory details as possible, and with a target of $45 \mathrm{~min}$ of exposure per session. In keeping with our prior VR treatment studies, a computer simulation of the 11th September WTC attacks was used to enhance an imaginal exposure therapy protocol (see Supplementary Materials and Methods and Difede et al, 2007). A psychologist who was blind to the medication condition conducted assessments at six time points: pre-treatment, following sessions $3,6,10$, immediately post-treatment, and 6 months later. All therapy sessions were videotaped, with a subset reviewed for protocol adherence. The study was registered with ClinicalTrials.gov (NCT00632632).

\section{Statistical Analysis}

Baseline characteristics of the treatment groups were compared using two-sided $t$-tests and $\chi^{2}$ analyses.

The primary outcome measure was total severity CAPS score, calculated by summing severity of each of the 17 PTSD symptoms. To calculate a diagnosis of PTSD according to DSM-IV criteria, the F1/I2 scoring rule, which requires a minimum score of 1 on the frequency scale and 2 on the intensity scale to qualify as a symptom, was used (Blake et al, 1998). The difference in CAPS scores was computed (relative to pre-treatment scores) and separate analysis of covariance were conducted at post-treatment and 6-month follow-up, with baseline scores entered as covariates. Controlled effect sizes (Hodges d) were computed for difference scores to assess the magnitude of effects. To analyze the rate of change at the interim assessments (after sessions 3,6, and 10), the mean percent change was compared between treatment groups at each interim time point (relative to pre-treatment) using twotailed $t$-tests. The rates of remission, defined as CAPS total score of $\leqslant 20$ and minimal or no impairment in social, occupational and other important areas of functioning (CAPS items F21 and F22 $\leqslant 1$ ), were compared using Fisher's exact test.

Ancillary analyses were conducted on SCID-MDD as well as on difference scores for CAPS cluster severity scores, CAPS sleep difficulty severity, PCL, BDI-II, and STAXI-2 


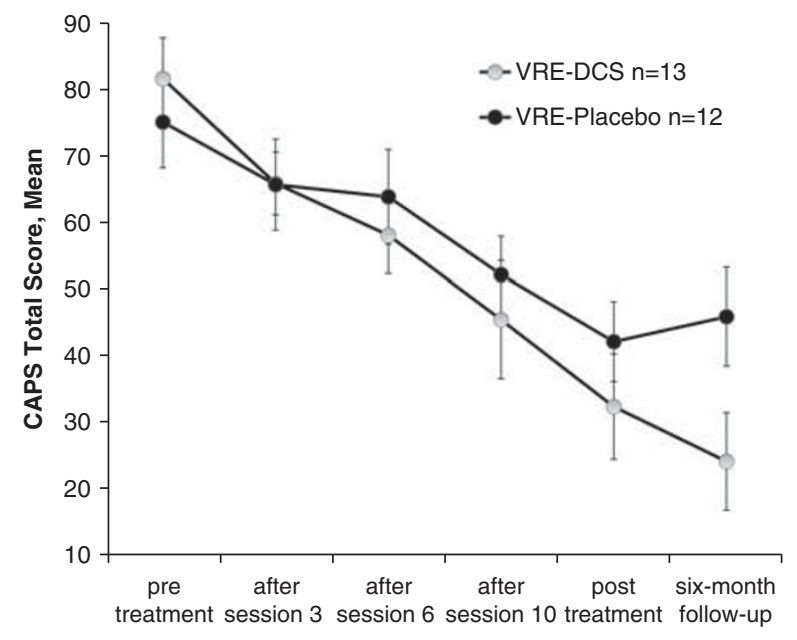

Figure I Mean CAPS severity scores over time.

severity scores (ANCOVAs). Concurrent medication use was coded using an indicator variable (use $v s$ no use) and its effect on change on the primary outcome variable was assessed using ANCOVA model.

All analyses adhered to intent-to-treat principle, with the last available observation used as the outcome data (ITT/ LOCF). Completer analysis was performed to evaluate robustness of study results. Ancillary analyses were not adjusted for the effect of multiple comparisons.

\section{RESULTS}

\section{Changes in Primary Outcome (CAPS)}

The difference between the VRE-DCS and VRE-placebo groups increased over time (Figure 1). Analyses of covariance on CAPS difference scores showed a pattern favoring the VRE-DCS group with a statistically nonsignificant medium effect at the conclusion of treatment $(F=2.42, \mathrm{df}=1,22, p=0.131$, Hodges $\mathrm{g}=0.68)$ and significant group differences at 6-month follow-up $(F=7.85, \mathrm{df}=1,22, p=0.010)$, with large effect size Hodges $g=1.13$ (Figure 2). Remission rates were significantly higher in the VRE-DCS group at both posttreatment $(6 / 13,46 \%$ vs $1 / 12,8 \%)$ and 6 -month follow-up (9/13, 69\% vs $2 / 12,17 \%)$.

Substantial between-groups difference in mean percent decrease in CAPS scores emerged at the interim assessment, which occurred after session 6 (VRE-DCS: $M=45.86$, $\mathrm{SD}=15.42 ; \quad$ VRE-placebo: $\mathrm{M}=29.59, \quad \mathrm{SD}=24.94 ; \quad t(23)$ $=-1.98, p=0.060$, 95\% CI $(-33.26,0.74)$. These gains were maintained through the 6-month follow-up (Table 2).

The pattern of results for PTSD clusters B, C, and D was similar to that observed for total CAPS severity score ( $p>0.05$ at post-treatment and $p<0.05$ at 6 -month followup, effect sizes cluster $\mathrm{B}: \mathrm{g}=0.53$ and $\mathrm{g}=0.88$; cluster $\mathrm{C}$ : $\mathrm{g}=0.56$ and $\mathrm{g}=1.15$; cluster $\mathrm{D}: \mathrm{g}=0.79$ and $\mathrm{g}=1.07)$. Further post-hoc analysis showed that the VRE-DCS group reported less sleep disturbance (as measured by CAPS sleep item) than the VRE-placebo group at the 6-month follow-up $(p=0.014, \mathrm{~g}=0.82$; Supplementary Figure S2).

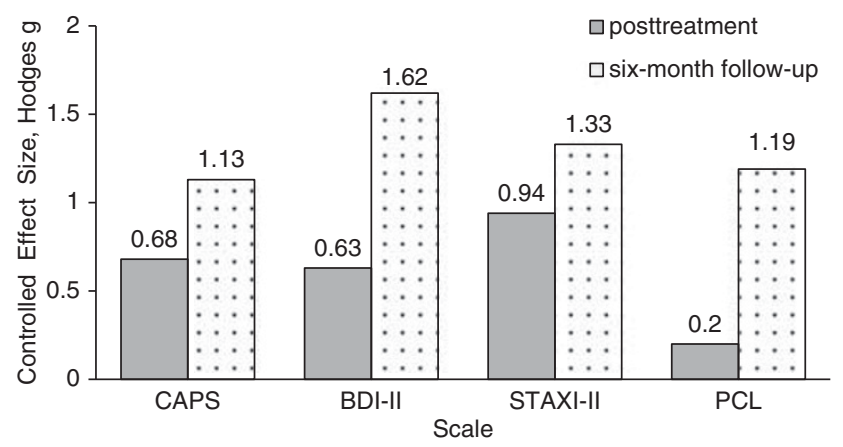

Figure 2 Controlled effect sizes (Hodges g) at post-treatment and 6month follow-up comparing VRE-DCS and VRE-placebo groups for primary and secondary outcome measures. Note: controlled effect sizes (Hodges g) cutoffs scores are: 0.2-small, 0.5-medium, and 0.8-large.

\section{Changes in Secondary Outcomes (SCID-MDD, BDI-II, STAXI-2, and PCL)}

The analysis of covariance on the BDI-II difference scores showed a trend at post-treatment and significant betweengroup effects at the 6-month follow-up $(F=3.74, \mathrm{df}=1,22$, $p=0.066$ and $F=19.78, \mathrm{df}=1,22, p<0.001$, respectively). Analysis of SCID major depressive disorder (SCID-MDD) data at post-treatment and 6-month follow-up further supported consistency in study results. Seven of nine participants in VRE-DCS who were diagnosed with MDD at baseline showed remission at post-treatment and an additional participant showed remission at the 6-month follow-up. Among study completers with MDD in the VREplacebo group $(n=5)$, three participants remitted at posttreatment but relapsed at the 6-month follow-up, one remitted at the 6-month follow-up, and one maintained MDD diagnosis at all three time-points. In addition, at the 6month follow-up there was a significant difference in STAXI2 anger expression index $(F=7.31, \mathrm{df}=1,21, p=0.009)$ (Figure 3 ) and PCL scores $(F=8.56, \mathrm{df}=1,22, p=0.008)$. Figure 2 presents effect sizes for all continuous secondary measures.

\section{Sensitivity Analysis}

Completer analysis included the 22 study completers ( $n=13$ in the VRE-DCS group and $n=9$ in the VREplacebo group) and yielded an identical patterns of results as compared with the ITT/LOCF analysis for both the primary outcome (CAPS) and all secondary outcomes (SCID-MDD, BDI-II, STAXI-2, and PCL). All three dropouts were in the VRE-placebo group and the observed differences in group mean were minimal for all measures (eg, VRE-placebo group CAPS scores at post-treatment: intentto-treat analysis: $\mathrm{M}=42.17, \mathrm{SD}=20.75$ vs completer analysis $\mathrm{M}=43.11, \mathrm{SD}=18.56$; at 6 -month follow-up: intent-to-treat analysis $\mathrm{M}=45.92, \mathrm{SD}=22.66$ vs completer analysis $\mathrm{M}=48.11, \mathrm{SD}=25.31$ ).

\section{Psychotropic Medication Use}

About half of participants $(n=14,56 \%)$ were on a stable dose of psychotropic medications ( $n=9$ in the VRE-DCS group, 
Table 2 Change in PTSD (CAPS) Severity Over Time

\begin{tabular}{|c|c|c|c|}
\hline & $\begin{array}{c}\text { VRE-DCS }(n=13) \\
\text { Mean (SD) }\end{array}$ & $\begin{array}{c}\text { VRE-placebo }(n=12) \\
\text { N Mean }(S D)\end{array}$ & $\begin{array}{l}P \text { Value }(95 \% \mathrm{Cl}) \\
\text { (Percent change) }\end{array}$ \\
\hline Pre-treatment & $81.62(22.13)$ & $75.08(23.80)$ & \\
\hline $3 \mathrm{~A}$ assessment & $65.84(16.99)$ & $65.67(23.65)$ & \\
\hline Percent change at $3 \mathrm{~A}$ & $16.05(2 \mid .72)$ & $9.99(28.31)$ & $p=0.552(-26.83,|4.7|)$ \\
\hline $6 \mathrm{~A}$ assessment & $58.07(20.69)$ & $63.83(24.70)$ & \\
\hline Percent change at $10 \mathrm{~A}$ & $55.94(16.54)$ & $41.69(21.27)$ & $p=0.073(-29.94,1.45)$ \\
\hline Post-treatment & $32.38(28.55)$ & $42.17(20.75)$ & \\
\hline Percent change at post-treatment & $61.92(25.88)$ & $42.89(22.45)$ & $p=0.063(-39.16,1.09)$ \\
\hline Six-month follow-up & $24.15(26.48)$ & $45.92(25.66)$ & \\
\hline
\end{tabular}

Percent change was calculated relative to pre-treatment.

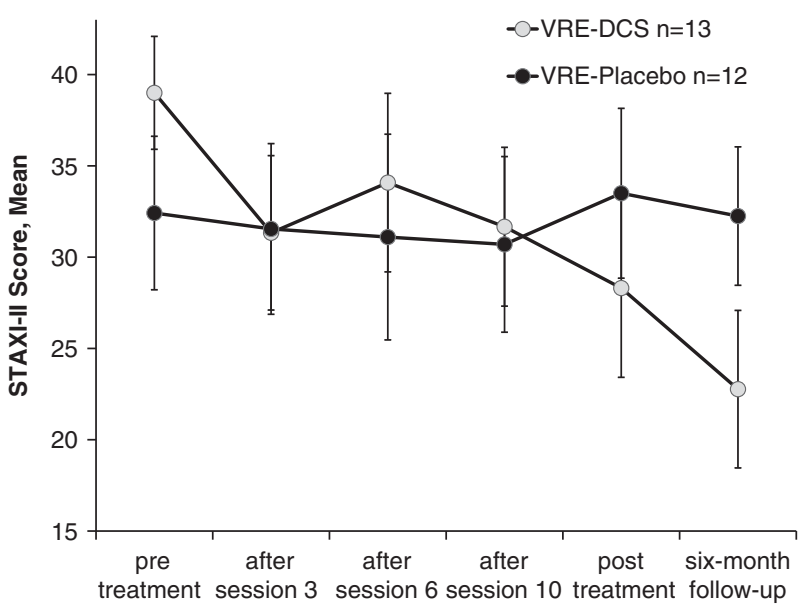

Figure 3 Mean STAXI-2 anger expression index over time.

$n=5$ in the VRE-placebo group, Table 1). Treatment gains were the largest in the VRE-DCS subgroup that did not use concurrent medication $(n=4)$, as compared with VRE-DCS subgroup on stable medications $(n=9)$ (CAPS change score at post-treatment $\mathrm{M}=61.50, \quad \mathrm{SD}=27.67 \quad$ vs $\mathrm{M}=43.78$, $\mathrm{SD}=21.45$; at the 6-month follow-up $\mathrm{M}=67.25, \mathrm{SD}=21.91$ vs $\mathrm{M}=54.00, \mathrm{SD}=18.65)$. The smallest gains were observed in the VRE-placebo group, regardless of concurrent medication use (no medications $n=7$ vs medication $n=5$, CAPS change score at post-treatment $\mathrm{M}=32.71, \mathrm{SD}=17.52$ vs $\mathrm{M}=33.20$, $\mathrm{SD}=30.26$; at the 6-month follow-up $\mathrm{M}=31.29, \mathrm{SD}=27.56 \mathrm{vs}$ $\mathrm{M}=26.20, \mathrm{SD}=33.62$ ). However, overall, the use of psychotropic medications did not have an effect on CAPS scores at either post-treatment $(F=1.67, \mathrm{df}=1,22, p=0.210)$ or the 6month follow-up $(F=1.67, \mathrm{df}=1,22, p=0.210)$.

\section{DISCUSSION}

Our results support the hypothesis that DCS enhances VRE therapy in the treatment of chronic PTSD. Patients in the
DCS-enhanced VRE group showed statistically and clinically significantly greater improvement in PTSD symptoms, as well as on secondary outcome measures of sleep, depression, and anger expression compared with the VREplacebo group. The findings were identical in the intent-totreat and completer samples. The medium to large effect sizes, across measures, at post-treatment and 6-month follow-up are particularly noteworthy given the small sample size. Patients in the VRE-DCS group showed greater improvement sooner than those in the VRE-placebo group. The VRE-DCS group also continued to improve on all measures after treatment ended. This is especially impressive given that both groups received an active psychotherapy with documented efficacy for the treatment of terroristrelated chronic PTSD (Difede et al, 2007; Parsons and Rizzo, 2008). In addition, PTSD symptom clusters B (re-experiencing), C (avoidance), and D (hyperarousal) in the VRE-DCS group showed significant improvement compared with the VRE-placebo group. Sleep disturbance, a salient cluster D symptom, is widely regarded as one of the most tenacious PTSD symptoms (Spoormaker and Montgomery, 2008). Thus, it is promising that those in the VRE-DCS group showed steady and significant improvement in sleep beginning midway through treatment and continuing through the 6-month follow-up.

Participant compliance was excellent. No significant medication side effects were reported, nor were dropouts related to medication side effects or inability to tolerate the VRE. Our treatment dropout rate of $12 \%$ is strikingly low in comparison with other PTSD treatment trials (Schottenbauer et al, 2008). Given the small number of dropouts, the fact that all dropouts were in the VRE-placebo group may be an artifact of the small sample size. However, it is also plausible that individuals in the VRE-DCS group were motivated to continue treatment because their symptoms remitted earlier than symptoms in the VRE-placebo group.

Both groups showed significant decreases in their PTSD symptoms compared with baseline, providing further evidence that VRE therapy is an effective treatment for chronic PTSD. Our data are consistent with earlier reports suggesting that VRE therapy is an effective treatment for WTC-related PTSD 
(Difede et al, 2007). VRE therapy is a particularly promising format to test the hypothesis that DCS enhances extinction learning, as sensory cues are standardized across patients. As the sensory cues in imaginal exposure are patient generated, and there are individual differences in the capacity to generate memorial imagery (Kosslyn et al, 2002), it is possible that DCS enhancement may be different for these two treatments. The use of VRE in this study may account for the differences in our findings compared with those of Litz et al (2012) who reported negative results suggesting that DCS did not enhance imaginal exposure for the treatment of PTSD, and de Kleine et al (2012) who showed a benefit of DCS for a subgroup of patients who had more severe pre-treatment PTSD.

The VRE therapy delivered sensory-rich cues obviating the need for the patient to engage in the effortful imaginal exposure that is so often difficult for patients who, by the nature of their symptoms, engage in avoidance of the recall of their traumatic memory. One could speculate that the sensoryrich stimuli may facilitate greater activation of the fear response in the amygdala, compared with imaginal exposure, allowing the DCS enhancement of extinction learning to occur. Moreover, by providing more and sensory-rich cues, which may match the patient's fear cues, the VRE treatment may also facilitate extinction learning simply by providing greater opportunity for DCS augmentation.

The robustness of our findings suggests that DCS may be seen as a novel pharmacological agent to augment and accelerate psychotherapeutic gains. As a partial agonist at the NMDA receptor, DCS purportedly acts by facilitating extinction learning - the mechanism of action hypothesized to underlie exposure therapy. These results are particularly noteworthy because the treatment paradigm utilized the acutely timed medication (ie, DCS) to enhance extinction learning, not to palliate the symptoms of PTSD. This stands in contrast to current and standard use of pharmacological agents in PTSD treatment, which have typically been offered to alleviate symptoms of insomnia, depression, and anxiety.

Given the small numbers of subjects in each treatment group taking concurrent psychotropic medications and the variation in medication among subjects, we are unable to draw any conclusions regarding a differential response to DCS associated with psychotropic medication usage. However, prior studies have reported that extinction enhancement following DCS was attenuated in rats pre-treated with 14 days of DCS or imipramine (Werner-Seidler and Richardson, 2007) and that chronic treatment with an SNRI or a tricyclic antidepressant downregulates NMDA receptor sensitivity in the cerebral cortex (Nowak et al, 1993) and frontal cortex and hippocampus (Calabrese et al, 2012; Bobula and Hess, 2008). These animal data suggest that the effect of DCS would be underestimated in humans taking these medications. Consistent with these pre-clinical studies, in our study treatment gains were the largest in the VRE-DCS subgroup that did not use concurrent medication, as compared with VRE-DCS subgroup on stable medications. The smallest gains were observed in the VRE-placebo group, regardless of concurrent medication use. Although our data are consistent with this pre-clinical data, our sample size is too small to draw any conclusions, which must await future studies with sample sizes sufficient for such analyses.

These pilot data add to the literature showing that DCSenhanced exposure therapy is effective for the treatment of anxiety disorders (Hofmann et al, 2006; Ressler et al, 2004). Our data, in conjunction with that of Ressler et al (2004), Hofmann et al (2006), and Otto et al (2010), form a compelling pattern of evidence supporting the hypothesis that DCS may facilitate extinction learning. The equivocal results reported in two recent PTSD studies may be accounted for by methodological issues. Litz et al (2012) reported negative results from a six-session protocol, which used a $50 \mathrm{mg}$ dose of DCS administered $30 \mathrm{~min}$ before the start of each exposure therapy session in a small sample of combat-related PTSD patients. de Kleine et al (2012) also used a $50 \mathrm{mg}$ dose given $1 \mathrm{~h}$ before the exposure session with a mixed trauma sample; half of whom reported PTSD secondary to childhood sexual assault. In this study, a $100 \mathrm{mg}$ dose of DCS was administered 90 min before the beginning of the treatment session. Thus, both the amount and timing of DCS may account for the differences in results. It is also possible that the DCS effect was blunted by the use of psychotropic medications, although this is speculative as an analysis of the influence of medication changes on the negative treatment outcome was not provided by Litz et al (2012). As OIF-OEF veterans are likely to have been exposed to multiple traumatic scenarios with a background of chronic threat during their tour of duty (Hoge et al, 2004), it is possible that the complexity of the traumas represented in the Litz et al (2012) study were not amenable to a brief therapy as compared with the relatively discrete time period of the WTC trauma.

These results warrant further study of DCS-enhanced exposure therapy for the treatment of chronic PTSD. The promising result regarding improvement in sleep functioning should be followed up with a comprehensive sleep assessment in future DCS studies. Studies are also needed to define the optimal timing of DCS administration and dosage, especially in light of differences in findings among the three PTSD-DCS studies to date.

Our results hold promise for reducing the distress and disability associated with PTSD. Patients in the DCS-enhanced VRE treatment group showed earlier, greater, and longer-lasting improvement compared with the VRE-placebo group. These data may be especially important for those in occupations-atrisk for PTSD (eg, firefighters, police officers, and soldiers), where an expeditious return to work is imperative. Use of DCS to enhance exposure therapy may not only improve social and occupational functioning, but also reduce the associated cost of disability, decreased productivity, and absenteeism.

\section{FUNDING AND DISCLOSURE}

Partial funding support was provided by DeWitt-Wallace Fund of the New York Community Trust, which had no role in study design, data collection, analysis and interpretation, or writing of this paper. On matters unrelated to this study, Dr Altemus has consulted for Ironwood Pharmaceuticals and Corcept Therapeutics, and has received research support from the Fisher Family Foundation. The authors declare no conflict of interest.

\section{REFERENCES}

Alonso J, Petukhova M, Vilagut G, Chatterji S, Heeringa S, Ustun TB et al (2011). Days out of role due to common physical and 
mental conditions: results from the WHO World Mental Health surveys. Mol Psychiatry 16: 1234-1246.

Baron H, Epstein IG, Mulinos MG, Nair KG (1956). Absorption, distribution, and excretion of cycloserine in man. Antibiotics Annual 16: 1234-1246.

Beck AT, Steer RA, Brown GK (1996). Manual for the Beck Depression Inventory-II. Psychological Corporation: San Antonio, TX, USA.

Blake DD, Weathers FW, Nagy LM, Kalopek DG, Charney DS, Keane TM (1998). Clinician-Administered PTSD Scale for DSM$I V$. Boston VA Medical Center, National Center for Posttraumatic Stress Disorder, Behavioral Science Division: Boston, MA, USA.

Bobula B, Hess G (2008). Antidepressant treatments-induced modifications of glutamatergic transmission in rat frontal cortex. Pharmacol Rep 60: 865-871.

Boje KM, Wong G, Skolnick P (1993). Desensitization of the NMDA receptor complex by glycinergic ligands in cerebellar granule cell cultures. Brain Res 603: 207-214.

Calabrese F, Guidotti G, Molteni R, Racagni G, Mancini M, Riva MA (2012). Stress-induced changes of hippocampal NMDA receptors: modulation by duloxetine treatment. PLoS One 7: e37916.

Davis M, Ressler K, Rothbaum BO, Richardson R (2006). Effects of $\mathrm{D}$-cycloserine on extinction: translation from preclinical to clinical work. Biol Psychiatry 60: 369-375.

de Kleine RA, Hendriks GJ, Kusters WJ, Broekman TG, van Minnen A (2012). A randomized placebo-controlled trial of D-cycloserine to enhance exposure therapy for posttraumatic stress disorder. Biol Psychiatry 71: 962-968.

Dedert EA, Calhoun PS, Watkins LL, Sherwood A, Beckham JC (2010). Posttraumatic stress disorder, cardiovascular, and metabolic disease: a review of the evidence. Ann Behav Med 39: 61-78.

Difede J, Cukor J, Jayasinghe N, Patt I, Jedel S, Spielman L et al (2007). Virtual reality exposure therapy for the treatment of posttraumatic stress disorder following September 11, 2001. J Clin Psychiatry 68: 1639-1647.

First MB, Spitzer RL, Williams JBW, Gibbon M (1997). Structured Clinical Interview for DSM-IV SCID. American Psychiatric Association: Washington, DC, USA.

Foa EB, Keane T, Friedman M (eds). (2000). Effective Treatments for PTSD: Practice guidelines from the International Society for Traumatic Stress Studies. Guilford Press: New York, NY, USA.

Foa EB, Kozak MJ (1986). Emotional processing of fear: exposure to corrective information. Psychol Bull 99: 20-35.

Guastella AJ, Richardson R, Lovibond PF, Rapee RM, Gaston JE, Mitchell P et al (2008). A randomized controlled trial of D-cycloserine enhancement of exposure therapy for social anxiety disorder. Biol Psychiatry 63: 544-549.

Hidalgo RB, Davidson JR (2000). Posttraumatic stress disorder: epidemiology and health-related considerations. J Clin Psychiatry 61(Suppl 7): 5-13.

Hofmann SG, Meuret AE, Smits JA, Simon NM, Pollack MH, Eisenmenger $\mathrm{K}$ et al (2006). Augmentation of exposure therapy with D-cycloserine for social anxiety disorder. Arch Gen Psychiatry 63: 298-304.

Hoge CW, Castro CA, Messer SC, McGurk D, Cotting DI, Koffman RL (2004). Combat duty in Iraq and Afghanistan, mental health problems, and barriers to care. N Engl J Med 351: 13-22.

Institute of Medicine (IOM) (2008). Treatment of Postraumatic Stress Disorder: An Assessment of the Evidence. The National Academies Press: Washington, DC, USA.
Kessler RC (2000). Posttraumatic stress disorder: the burden to the individual and to society. J Clin Psychiatry 61(Suppl 5): 4-12 discussion 13-14.

Kessler RC, Sonnega A, Bromet E, Hughes M, Nelson CB (1995). Posttraumatic stress disorder in the National Comorbidity Survey. Arch Gen Psychiatry 52: 1048-1060.

Kosslyn SM, Cacioppo JT, Davidson RJ, Hugdahl K, Lovallo WR, Spiegel D et al (2002). Bridging psychology and biology: the analysis of individuals in groups. Am Psychol 57: 341-351.

Ledgerwood L, Richardson R, Cranney J (2003). Effects of D-cycloserine on extinction of conditioned freezing. Behav Neurosci 117: 341-349.

Litz BT, Salters-Pedneault K, Steenkamp MM, Hermos JA, Bryant RA, Otto MW et al (2012). A randomized placebo-controlled trial of D-cycloserine and exposure therapy for posttraumatic stress disorder. J Psychiatr Res 46: 1184-1190.

Nowak G, Trullas R, Layer RT, Skolnick P, Paul IA (1993). Adaptive changes in the N-methyl-D-aspartate receptor complex after chronic treatment with imipramine and 1-aminocyclopropanecarboxylic acid. J Pharmacol Exp Ther 265: 1380-1386.

Otto MW, Tolin DF, Simon NM, Pearlson GD, Basden S, Meunier SA et al (2010). Efficacy of D-cycloserine for enhancing response to cognitive-behavior therapy for panic disorder. Biol Psychiatry 67: $365-370$.

Parsons TD, Rizzo AA (2008). Affective outcomes of virtual reality exposure therapy for anxiety and specific phobias: a meta analysis. J Behav Ther Exp Psychiatry 39: 250-261.

Quartermain D, Mower J, Rafferty MF, Herting RL, Lanthorn TH (1994). Acute but not chronic activation of the NMDA-coupled glycine receptor with $\mathrm{D}$-cycloserine facilitates learning and retention. Eur J Pharmacol 257: 7-12.

Ressler KJ, Rothbaum BO, Tannenbaum L, Anderson P, Graap K, Zimand E et al (2004). Cognitive enhancers as adjuncts to psychotherapy: use of D-cycloserine in phobic individuals to facilitate extinction of fear. Arch Gen Psychiatry 61: 1136-1144.

Schottenbauer MA, Glass CR, Arnkoff DB, Tendick V, Gray S (2008). Nonresponse and dropout rates in outcome studies on PTSD: review and methodological considerations. Psychiatry Interpersonal Biological Processes 71: 134-168.

Spielberger C (1999). STAXI-2 State-Trait Anger Expression Inventory-2: Professional Manual. Psychological Assessment Resources: Odessa, FL, USA.

Spoormaker VI, Montgomery P (2008). Disturbed sleep in posttraumatic stress disorder: secondary symptom or core feature? Sleep Med Rev 12: 169-184.

Walker DL, Ressler KJ, Lu KT, Davis M (2002). Facilitation of conditioned fear extinction by systemic administration or intraamygdala infusions of D-cycloserine as assessed with fearpotentiated startle in rats. J Neurosci 22: 2343-2351.

Weathers FW, Litz FW, Herman DS, Huska JA, Keane TM (1993). The PTSD Checklist (PCL): reliability, validity, and diagnostic utility. Annual Meeting of the International Society for Traumatic Stress Studies. San Antonio, TX, USA.

Werner-Seidler A, Richardson R (2007). Effects of D-cycloserine on extinction: consequences of prior exposure to imipramine. Biol Psychiatry 62: 1195-1197.

Yule W (2001). Posttraumatic stress disorder in the general population and in children. J Clin Psychiatry 62(Suppl 17): 23-28.

Zimmerman JM, Maren S (2010). NMDA receptor antagonism in the basolateral but not central amygdala blocks the extinction of Pavlovian fear conditioning in rats. Eur J Neurosci 31: $1664-1670$.

Supplementary Information accompanies the paper on the Neuropsychopharmacology website (http://www.nature.com/npp) 